\title{
Assessing acquired resistance to IDH1 inhibitor therapy by full-exon IDH1 sequencing and structural modeling
}

\author{
Zoltán N. Oltvai, ${ }^{1,2,3,4,7}$ Susan E. Harley, ${ }^{1,5,7}$ David Koes, ${ }^{2}$ Stephen Michel, ${ }^{1}$ \\ Erica D. Warlick, ${ }^{6}$ Andrew C. Nelson, ${ }^{1}$ Sophia Yohe, ${ }^{1}$ and Pawel Mroz ${ }^{1}$ \\ ${ }^{1}$ Department of Laboratory Medicine and Pathology, University of Minnesota, Minneapolis, Minnesota 55455, \\ USA; ${ }^{2}$ Department of Computational and Systems Biology, University of Pittsburgh, Pittsburgh, Pennsylvania \\ 15260, USA; ${ }^{3}$ Department of Pathology, University of Pittsburgh, Pittsburgh, Pennsylvania 15261, USA; \\ ${ }^{4}$ Department of Pathology and Laboratory Medicine, University of Rochester, Rochester, New York 14642, \\ USA; ${ }^{5}$ Division of Hematology, Oncology, and Transplantation, ${ }^{6}$ Department of Medicine, University of \\ Minnesota, Minneapolis, Minnesota 55455, USA
}

\begin{abstract}
Somatic mutations in hotspot regions of the cytosolic or mitochondrial isoforms of the isocitrate dehydrogenase gene (IDH1 and IDH2, respectively) contribute to the pathogenesis of acute myeloid leukemia (AML) by producing the oncometabolite 2-hydroxyglutarate (2-HG). The allosteric IDH1 inhibitor, ivosidenib, suppresses 2-HG production and induces clinical responses in relapsed/refractory IDH1-mutant AML. Herein, we describe a clinical case of AML in which we detected the neomorphic IDH1 p.R132C mutation in consecutive patient samples with a mutational hotspot targeted next-generation sequencing (NGS) assay. The patient had a clinical response to ivosidenib, followed by relapse and disease progression. Subsequent sequencing of the relapsed sample using a newly developed all-exon, hybrid-capture-based NGS panel identified an additional IDH1 p.S280F mutation known to cause renewed 2-HG production and drug resistance. Structural modeling confirmed that serine-to-phenylalanine substitution at this codon sterically hinders ivosidenib from binding to the mutant IDH1 dimer interface and predicted a similar effect on the pan-IDH inhibitor AG-881. Joint full-exon NGS and structural modeling enables monitoring IDH1 inhibitor-treated AML patients for acquired drug resistance and choosing follow-up therapy.
\end{abstract}

Corresponding author: pmroz@umn.edu

(c) 2021 Oltvai et al. This article is distributed under the terms of the Creative Commons Attribution-NonCommercial License, which permits reuse and redistribution, except for commercial purposes, provided that the original author and source are credited.

Ontology term: leukemia

Published by Cold Spring Harbor Laboratory Press

doi:10.1101/mcs.a006007
[Supplemental material is available for this article.]

\section{INTRODUCTION}

Molecular characterization of acute myeloid leukemia (AML) yielded greater understanding of leukemogenesis, revolutionized disease diagnosis and prognosis, and led to the development of new biomarkers and novel targeted therapies (King and Bagg 2017; Carbonell et al. 2019; Winer and Stone 2019; DiNardo and Wei 2020). Somatic mutations in two isoforms of isocitrate dehydrogenase gene (IDH1/2) characterize 20\%-25\% of AML cases (Losman et al. 2013; Nassereddine et al. 2017; Yoshimi et al. 2019). IDH1/2 are homodimer enzymes that normally catalyze the conversion of isocitrate to a-ketoglutarate (aKG) (Tommasini-Ghelfi et al. 2019). Oncogenic IDH mutations map to key structural arginine residues within these enzymes' active site that are critical for isocitrate binding: the amino acid (AA) R132 in IDH1 and the AA R140 or

\footnotetext{
${ }^{7}$ These authors contributed equally to this work.
} 
COLD SPRING HARBOR Molecular Case Studies
Assessment of acquired IDH1 inhibitor resistance

$\mathrm{R} 172$ in IDH2. These mutations cause conversion of the physiologic metabolite aKG into (R) 2hydroxyglutarate (2-HG) through theirneomorphic activity (Dang et al. 2010, 2016; Losman and Kaelin 2013). 2-HG acts as an oncometabolite by competitively inhibiting aKG-dependent histone demethylases, prolyl hydroxylases, and TET hydroxylases, which in turn alters the epigenome and inhibits normal cellular differentiation (Medeiros et al. 2017).

Targeted inhibition of pathogenic IDH1/2 variants has been shown to reduce the production of 2-HG and decrease leukemic burden (Chaturvedi et al. 2013; Wang et al. 2013; Upadhyay et al. 2017; Yen et al. 2017). Small-molecule inhibitors enasidenib (Celgene Corp. AG-221/CC-9007) and ivosidenib (Agios Pharmaceuticals, Inc. AG-120) have been approved by the U.S. Food and Drug Administration (FDA) for the treatment of IDH-mutated AML. The pan-IDH inhibitor AG-881 is being studied in clinical trials (Nassereddine et al. 2017). Ivosidenib in monotherapy has achieved complete remission (CR) in $24 \%$ of cases, an overall response of $42 \%$, and a median overall survival of 9 mo in patients with relapsed/refractory (R/R) IDH1 mutant AML (Megías-Vericat et al. 2019). However, reports have already emerged describing cases of relapse during therapy and acquired resistance to these mutant IDH1/2 inhibitors (Harding et al. 2018; Intlekofer et al. 2018; Quek et al. 2018; Choe et al. 2020).

Herein, we describe a patient with R/R AML in whom we identified the neomorphic p.R132C IDH1 mutation by using hotspot amplicon-based next-generation sequencing (NGS). Initially, the patient showed response to ivosidenib therapy, but relapsed $\sim 8$ mo after start of treatment. Complete IDH1 exon sequencing using a newly developed hybrid-capture NGS assay detected an IDH1 p.S280F variant in addition to the known pathogenic p.R132C variant. Retrospective testing of all available previous patient samples confirmed the absence of a second-site p.S280F mutation prior to ivosidenib therapy. Structural modeling showed that the p.S280F variant is located at the ivosidenib binding site on the interface of the IDH1 dimer. Modeling also predicted steric hindrance with the substitution of phenylalanine for serine that would interfere with binding of both ivosidenib and the panIDH inhibitor, AG-881, excluding the latter as potential follow-up therapy for AML cases bearing these mutations.

\section{RESULTS}

\section{Case Presentation}

A 68-yr-old female initially presented in 2016 to an outside hospital with a diagnosis of myelodysplastic syndrome with excess blasts (MDS-EB-2). Despite treatment with hypomethylating agents, her disease progressed to AML with myelodysplasia-related changes (AML-MRC) positive for FLT3 internal tandem duplication (FLT3-ITD) (that was identified at an outside laboratory) in 2017. She was initially treated by her community oncologist with two cycles of cytarabine, but because her AML persisted she was reinduced with cytarabine, daunorubicin, and midostaurin in early January 2018. Subsequent bone marrow biopsy demonstrated morphologic remission and the patient underwent consolidation therapy with idarubicin in combination with cytarabine (IDAC) and midostaurin. Thereafter, she was referred to the University of Minnesota for possible stem cell transplantation.

Restaging bone marrow biopsy in late February 2018 showed persistent/recurrent lowlevel disease. At this time, an amplicon-based NGS test was negative for the previously identified FLT3-ITD mutation but detected an IDH1 p.R132C variant (Supplemental Fig. S1). The patient was subsequently treated with combination of venetoclax and cytarabine; however, restaging bone marrow biopsy in July 2018 demonstrated persistent/recurrent leukemia with 77\% circulating blasts (data not shown). In August 2018, targeted treatment was initiated with the then newly FDA-approved IDH1 inhibitor, ivosidenib. A follow-up bone marrow biopsy in December 2018 showed morphologic remission (Fig. 1A). However, flow cytometry 

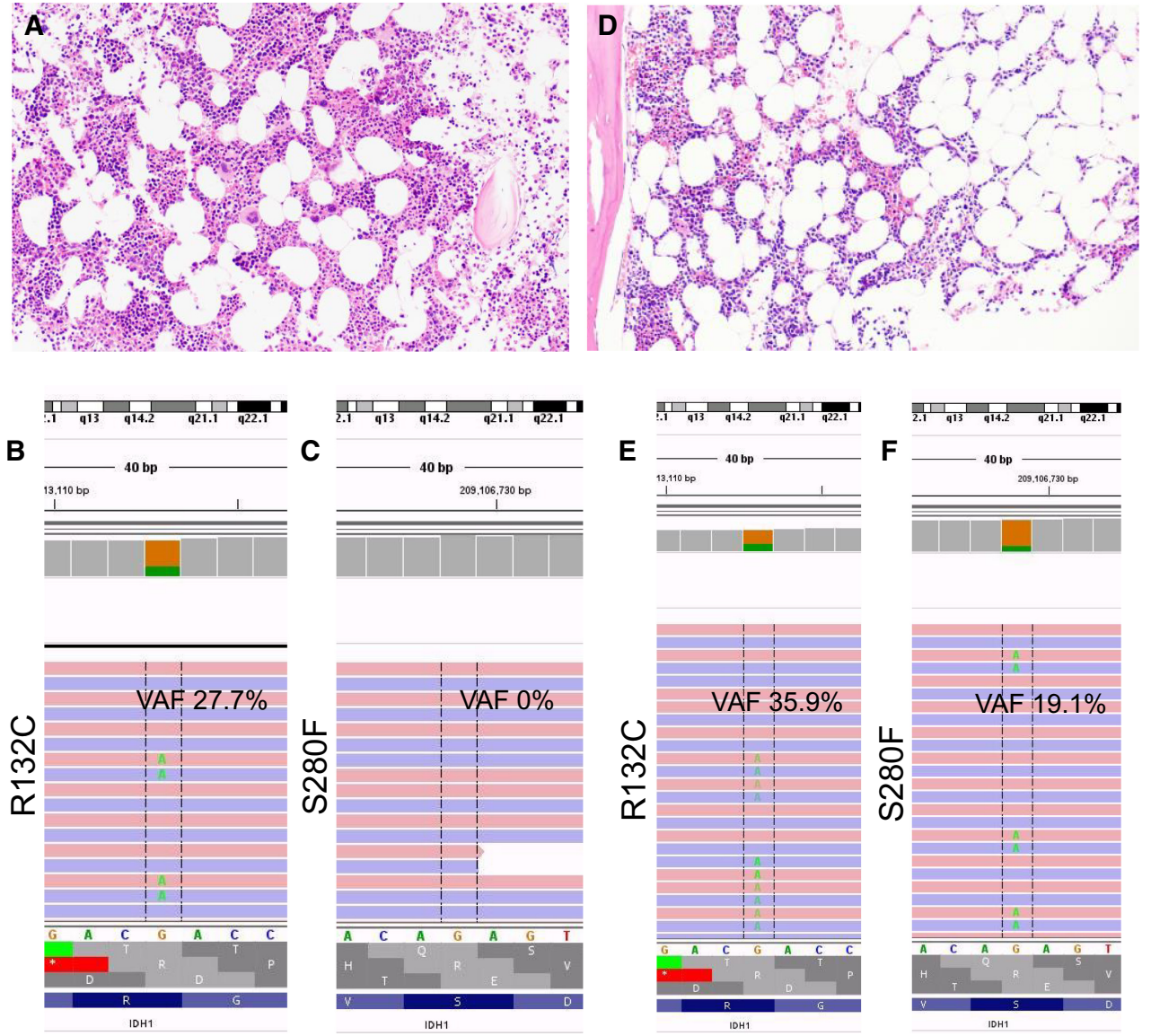

Figure 1. Representative morphologic, immunophenotyping, and IDH1 mutation data. Bone marrow biopsies from December 2018 (A) and March 2019 (D). The December 2018 biopsy, performed 4 mo after initiation of IDH1 inhibitor therapy, showed trilineage hematopoiesis (A), with up to $5 \%$ blasts by manual differential ( 500 cell count) and $1 \%$ by flow cytometry (IFC) (Supplemental Fig. S2A). NGS data showed persistence of the IDH1 p.R132C mutation (B); a p.S280F mutation was not detectable (C). The follow-up biopsy in March 2019, performed 8 mo into IDH1 inhibitor therapy, showed recurrent AML with 44\% abnormal myeloblasts by manual differential and core biopsy demonstrating clusters and small sheets of blasts (D), and with $49 \%$ blasts by flow cytometry (IFC) (Supplemental Fig. S2B). NGS detected the presence of the IDH1 p.R132C variant (E) as well as an additional new IDH1 p.S280F variant $(F)$. In $B, C, E$, and $F$, the IGV screenshots of the hybrid capture-based NGS results are shown. (VAF) Variant allele frequency.

immunophenotyping was concerning for low-level disease with persistence of partial CD7 expression (Supplemental Fig. S2A). A concurrent amplicon-based NGS test showed persistence of the IDH1 p.R132C variant (Fig. 1B). Overall, these findings were consistent with remission but concerning for minimal residual disease.

Ivosidenib therapy was continued with stable peripheral blood counts until March 2019, when the patient represented with leukemia relapse. Morphology showed recurrent AML with $44 \%$ abnormal myeloblasts by manual differential and core biopsy demonstrating clusters and small sheets of blasts (Fig. 1D), and with 49\% blasts by flow cytometry with persistence of partial CD7 expression (Supplemental Fig. S2B). A newly validated hybridcapture-based NGS assay again detected the p.R132C variant in the relapse sample (Fig. 1E) alongside an additional IDH1 p.S280F variant (Fig. 1F). Concurrent cytogenetics and fluorescence in situ hybridization (FISH) studies showed a complex karyotype, including 
previously detected monosomy 7 (Supplemental Table S1). These are poor prognostic indicators that likely contributed to this patient's progression/relapse. Two months after relapse, the patient died as a result of pseudomonal bacteremia and sepsis.

\section{Amplicon-Based NGS Testing}

We performed amplicon-based NSG studies on bone marrow aspirate samples as part of the patient's routine clinical care. The assay tested for hotspot mutations in the following genes: FLT3, NPM1, KIT, IDH1, IDH2, NRAS, KRAS, PDGFR, TP53, and WT1 (Henzler et al. 2018) (see Methods). We identified no pathogenic variants in the diagnostic sample. We first detected the IDH1 p.R132C mutation in the sample from February 2018. This mutation remained persistently detectable throughout her treatment course to the final March 2019 test (Table 1) at disease relapse. Of note, the persistence of IDH1/2 mutations in AML patients is known to be significant for predicting relapse (Ok et al. 2019).

\section{Hybrid-Capture-Based NGS Retesting of all Patient Samples}

We used a newly developed and clinically validated hybrid-capture-based NGS assay to test the bone marrow aspirate sample at the time of the patient's relapse in March 2019. The

\begin{tabular}{|c|c|c|c|c|c|c|c|c|c|}
\hline Sample ID & & $16-04470$ & $18-01654$ & $18-02858$ & $18-04274$ & $18-05644$ & $18-08274$ & $18-10037$ & $19-01827$ \\
\hline Date & & $6 / 8 / 2016$ & $2 / 28 / 2018$ & $\begin{array}{l}4 / 10 / \\
2018\end{array}$ & $5 / 30 / 2018$ & $7 / 13 / 2018$ & 10/8/2018 & $12 / 6 / 2018$ & 2/28/2019 \\
\hline Time point & & $\begin{array}{r}\text { Diagnosis } \\
\text { of MDS }\end{array}$ & $\begin{array}{l}1.5 \text { mo post } \\
\text { induction } \\
\text { for AML }\end{array}$ & $\begin{array}{c}4 \text { mo post } \\
7+3 \\
\text { therapy }\end{array}$ & $\begin{array}{l}\text { Post } \\
\text { cytarabine/ } \\
\text { venetoclax }\end{array}$ & $\begin{array}{c}\text { Post } 2 \text { cycles } \\
\text { cytarabine/ } \\
\text { venetoclax }\end{array}$ & $\begin{array}{l}\text { Recent } \\
\text { IDH1 } \\
\text { inhibitor } \\
\text { therapy }\end{array}$ & $\begin{array}{c}4 \text { mo IDH1 } \\
\text { inhibitor } \\
\text { therapy }\end{array}$ & $\begin{array}{c}7 \text { mo IDH1 } \\
\text { inhibitor } \\
\text { therapy }\end{array}$ \\
\hline $\begin{array}{c}\text { Morphologic } \\
\text { diagnosis }\end{array}$ & & MDS & $\begin{array}{l}\text { Probable } \\
\text { residual } \\
\text { MN }\end{array}$ & $\begin{array}{l}\text { Persistent } \\
\text { MN }\end{array}$ & $\begin{array}{l}\text { Probable } \\
\text { residual } \\
\text { MN }\end{array}$ & Residual MN & $\begin{array}{l}\text { Persistent } \\
\text { AML }\end{array}$ & Remission & AML \\
\hline $\begin{array}{l}\text { Blast \% } \\
\text { morph }\end{array}$ & & $8 \%$ & $4.50 \%$ & $3 \%$ & $4.60 \%$ & $\begin{array}{l}\text { Focal } \\
\text { increased }\end{array}$ & $5.00 \%$ & $0.60 \%$ & $44 \%$ \\
\hline Blast \% flow & & $4.70 \%$ & $4.50 \%$ & $7 \%$ & $2 \%$ & $<1 \%$ & $2 \%$ & $1 \%$ & $49 \%$ \\
\hline Gene & $\begin{array}{l}\text { Protein } \\
\text { change }\end{array}$ & VAF & VAF & VAF & VAF & VAF & VAF & VAF & VAF \\
\hline IDH1 & $\mathrm{R} 132 \mathrm{C}$ & 0.136 & 0.074 & 0.051 & 0.05 & 0.098 & 0.185 & 0.277 & 0.359 \\
\hline $\mid D H 1$ & $\mathrm{~S} 280 \mathrm{~F}$ & $\mathrm{Neg}$ & $\mathrm{Neg}$ & $\mathrm{Neg}$ & $\mathrm{Neg}$ & Neg & $\mathrm{Neg}$ & $\mathrm{Neg}$ & 0.191 \\
\hline FLT3 & ITD & Neg & $\mathrm{Neg}$ & $\mathrm{Neg}$ & $\mathrm{Neg}$ & Neg & $\mathrm{Neg}$ & $\mathrm{Neg}$ & $\mathrm{Neg}$ \\
\hline$B C O R$ & K1721Rfs*4 & $\mathrm{Neg}$ & $\mathrm{Neg}$ & $\mathrm{Neg}$ & $\mathrm{Neg}$ & $\mathrm{Neg}$ & 0.131 & $\mathrm{Neg}$ & $\mathrm{Neg}$ \\
\hline DNMT3A & L650Pfs*52 & 0.093 & 0.063 & 0.051 & $0.05^{*}$ & 0.074 & 0.131 & Neg* & 0.127 \\
\hline DNMT3A & $\mathrm{R} 899 \mathrm{H}$ & 0.195 & 0.206 & 0.212 & 0.181 & 0.12 & 0.166 & 0.356 & 0.358 \\
\hline GATA2 & L359S & $\mathrm{Neg}$ & Neg & Neg & Neg & Neg & 0.109 & Neg & 0.077 \\
\hline KRAS & G12A & Neg & Neg & Neg & $\mathrm{Neg}$ & $\mathrm{Neg}$ & 0.064 & $\mathrm{Neg}$ & 0.076 \\
\hline SF1 & G323R & $\mathrm{Neg}$ & $\mathrm{Neg}$ & $\mathrm{Neg}$ & $\mathrm{Neg}$ & $\mathrm{Neg}$ & 0.064 & $\mathrm{Neg}$ & 0.059 \\
\hline
\end{tabular}

Presence of mutations at various time points during the patient's disease course are shown, as detected by the full-exon, heme hybrid capture NGS assay. The IDH1 p.R132C mutation is highlighted in bold. It is evident that some variants, including likely germline variants, were maintained throughout the patient's disease course, whereas others appeared/disappeared as a result of clonal drift and/or clonal evolution, in part in response to drug therapies.

(mo) Month, (MDS) myelodysplastic syndrome, (MN) myeloid neoplasm, (AML) acute myeloid leukemia, (VAF) variant allele frequency, (ND) not done, (Neg) negative, (*) present in IGV but not Genelnsight after filter. 
assay enables the analysis of all coding exons of genes instead of just hotspot regions, and this assay's panel includes an expanded number of genes compared to our amplicon-based assay (see Methods for details). We detected the IDH1 p.R132C variant that previously characterized this patient's myeloid neoplasm, a pathogenic variant in the KRAS gene, p.G12A, and a pathogenic variant in the DNMT3A gene, p.L650Pfs*52. Importantly, we also identified a second IDH1 variant, p.S280F, in the relapse sample (Fig. 1F). IDH1 p.S280F (NM_005896.3: c.839C > T) is a missense variant described in two heterozygotes and no homozygotes in the gnomAD population database (https://gnomad.broadinstitute.org/; Karczewski et al. 2020) (accessed 09/25/2020). It is a rare variant absent from the COSMIC (https://cancer.sanger.ac.uk/cosmic; Forbes et al. 2008) and ClinVar (https://www.ncbi.nlm .nih.gov/clinvar/; Landrum et al. 2016) databases (accessed 09/25/2020). Based on lack of available evidence and the fact that this variant is not common in the general population, we initially classified it as a Tier III variant of unknown clinical significance (Li et al. 2017). However, we were aware that this variant had been described by Intlekofer et al. (2018) in a patient with acquired resistance to IDH1 inhibitor therapy.

We then used hybrid-capture NGS to test all available historical patient samples retrospectively to assess when during the patient's disease course this IDH1 variant emerged. The results confirmed that the patient consistently tested positive for the IDH1 p.R132C and DNMT3A p.L650Pfs*52 variants throughout her illness (Table 1). The KRAS p.G12A mutation was only seen in October 2018 and in the relapse sample. We also observed variants of uncertain significance either at a single time point (BCOR p.K1721Rfs*4, GATA2 p.L359S, and SF1 p.G323R genes) or in all samples (DNMT3A p.R899H) (see Table 1 for a full list of genes harboring variations in the context of clinical setting and pathology data, Table 2 for the details on the observed variants, and Supplemental Table S1 for concurrent cytogenetic findings). Significantly, the IDH1 p.S280F variant was only present in the post-ivosidenib therapy relapse sample (Fig. 1C,F; Supplemental Figs. S1D, S3). This suggests clonal evolution during the course of the disease under the selection pressure of IDH1 inhibitor therapy.

\section{Structural Basis for Ivosidenib Resistance}

Using structural modeling, we located the IDH1 p.S280F missense variant at the IDH1 dimer interface, as previously described (and at a position analogous to the IDH2 P.I319M variant in

\begin{tabular}{|c|c|c|c|c|c|c|c|}
\hline Gene & Chromosome & HGVS DNA reference & $\begin{array}{l}\text { HGVS protein } \\
\text { reference }\end{array}$ & $\begin{array}{l}\text { Variant } \\
\text { type }\end{array}$ & Predicted effect & $\begin{array}{l}\mathrm{dbSNP} / \mathrm{db} \text { Var } \\
\text { ID }\end{array}$ & $\begin{array}{l}\text { ClinVar } \\
\text { ID }\end{array}$ \\
\hline IDH1 & 2 & NM_005896.2 c.394C> T & p.R132C & SNV & Substitution & rs121913499 & 375891 \\
\hline IDH1 & 2 & NM_005896.2 c.839C > T & p.S280F & SNV & Substitution & rs757389021 & 996127 \\
\hline FLT3 & 13 & NM_004119.2 & & ITD & Insertion & & \\
\hline$B C O R$ & 2 & NM_001123385.1 c.5162delA & p.K1721Rfs*4 & Indel & $\begin{array}{l}\text { Deletion, } \\
\text { frameshift } \\
\text { termination }\end{array}$ & & \\
\hline DNMT3A & 2 & $\begin{array}{l}\text { NM_022552.5 } \\
\text { c.1949_1963delinsCCAAG }\end{array}$ & p.L650Pfs*52 & $\begin{array}{l}\text { Complex } \\
\text { indel }\end{array}$ & $\begin{array}{l}\text { Frameshift } \\
\text { termination }\end{array}$ & & \\
\hline DNMT3A & 2 & NM_022552.4 c.2696G >A & p.R899H & SNV & Substitution & - & - \\
\hline GATA2 & 3 & NM_032638.4 c.1076T > C & p.L359S & SNV & Substitution & - & - \\
\hline KRAS & 12 & NM_033360.3 c.35G >C & p.G12A & SNV & Substitution & rs121913529 & 45122 \\
\hline SF1 & 11 & NM_001178030.2 c.967G > A & p.G323R & SNV & Substitution & - & - \\
\hline
\end{tabular}

(ITD) Internal tandem duplication, (SNP) single-nucleotide polymorphism, (SNV) single-nucleotide variant. 
COLD SPRING HARBOR Molecular Case Studies
Assessment of acquired IDH1 inhibitor resistance
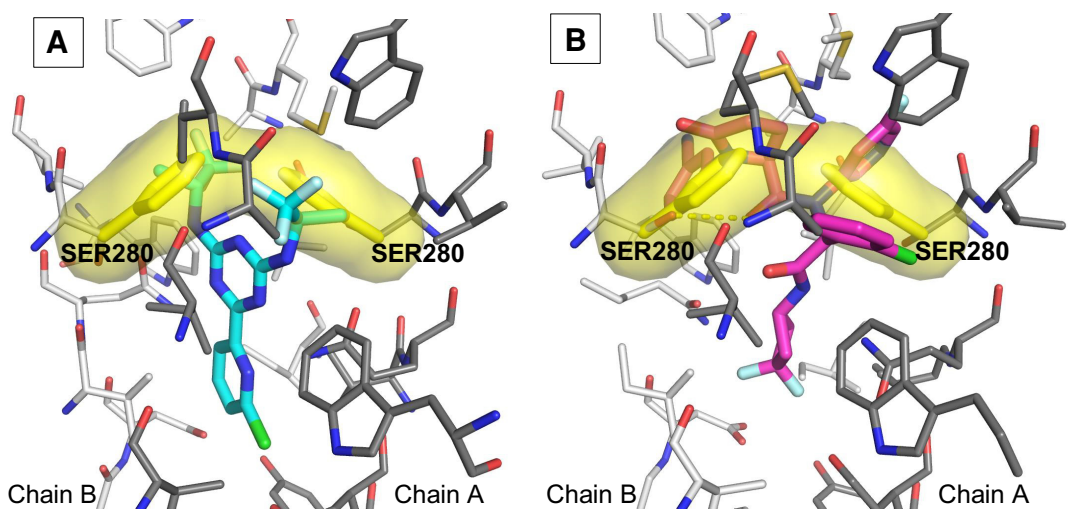

Figure 2. Crystal structure and modeling the IDH1 inhibitor binding site. (A) Crystal structure of IDH1 p.R132H mutant complexed with pan-IDH inhibitor, AG-881 (cyan) (PDB 6ADG) shown with superimposed phenylalanine mutations of serine (SER) 280 (yellow). (B) IDH1 inhibitor, ivosidenib (AG-120) (purple) docked to the same structure demonstrates an even greater incompatibility with the p.S280F mutation, including steric hindrance and the loss of hydrogen bonding to SER280.

a patient with acquired resistance to IDH2 inhibitor, enasidenib, therapy with increased 2HG levels [Intlekofer et al. 2018; Choe et al. 2020]). The structure of the dual IDH1/IDH2 inhibitor, AG-881 (also developed by Agios Pharmaceuticals) has a solved co-crystal with IDH1 p.R132H mutant (PDB 6ADG) (Supplemental Fig. S4; Ma and Yun 2018). This allosteric inhibitor and other IDH1-specific inhibitors bind at the dimer interface of IDH1 (Xie et al. 2017; Ma and Yun 2018; Waitkus et al. 2018). As shown in Figure 2A, mutating the amino acid serine 280 (SER280) in silico to phenylalanine results in significant steric clashes that are predicted to prevent the binding of AG-881. Hypothesizing that ivosidenib has a similar binding mode, we docked ivosidenib into this binding site (see Methods). The resulting ivosidenib pose makes polar contacts with the hydroxyl group of SER280 (Supplemental Fig. S5). These polar contacts are lost with phenylalanine substitutions. Therefore, ivosidenib is predicted to be even more sterically hindered by the p.S280F mutation than AG-881 (Fig. 2B). We note that the location of SER280 in IDH1 is identical, relative to the inhibitor and dimer interface, to the locations of the p.Q316E and p.I319M mutations in IDH2, which result in resistance to the IDH2 inhibitor, enasidenib (Intlekofer et al. 2018). However, the p.S280F mutation may have less or no impact on inhibitors that have different binding modes to IDH1, such as those targeting the active site (Jakob et al. 2018) or alternative allosteric sites (Levell et al. 2017) of IDH1.

\section{DISCUSSION}

AML is a genetically heterogeneous disease that is characterized by multiple somatically acquired mutations that affect genes of different functional categories, leading to a complex clonal architecture and disease evolution over time (Bullinger et al. 2017; Martignoles et al. 2018). Mutations in genes encoding epigenetic modifiers such as DNA (cytosine-5)methyltransferase 3A (DNMT3A), additional sex comb-like 1 (ASXL1), Tet methylcytosine dioxygenase 2 (TET2), and IDH1/2 are commonly acquired early in the disease course and are usually present in the founding clone. In contrast, signaling pathway mutations such as those affecting KRAS or FLT3 genes are often late genetic events (Bullinger et al. 2017; Martignoles et al. 2018). Such mutations can persist after therapy, lead to clonal expansion during hematologic remission, and eventually cause relapse (Bullinger et al. 
2017). IDH1 and IDH2 gene mutations are observed in up to $10 \%$ and $15 \%$ of AML cases, respectively. The majority of $I D H 2$ variants are missense mutations located at $A A$ arginine 140 (R140), whereas the majority of IDH1 variants are cysteine substitutions at AA position R132 (Chotirat et al. 2012). Recently, the FDA approved two new targeted therapeutics that inhibit mutated IDH1 and IDH2, ivosidenib (AG-120) and enasidenib (AG-221), respectively, for treating R/R AML (Golub et al. 2019). Ongoing studies have shown that these agents are relatively well-tolerated. Ivosidenib has shown variable efficacy as monotherapy, ranging from complete to partial remission in patients with R/R AML (Megías-Vericat et al. 2019). However, some of these patients have experienced disease recurrence after treatment.

Recent studies have identified several post-therapy resistance mechanisms in response to IDH1 or IDH2 inhibitor therapies. These include clonal evolution, the emergence of secondary mutations in receptor tyrosine kinase pathway and RAS family members, and selection of terminal or ancestral clones (Amatangelo et al. 2017; DiNardo et al. 2018; Quek et al. 2018; Choe et al. 2020). In addition, isoform switching from mutant IDH1 to mutant IDH2 or vice versa (Harding et al. 2018) or the appearance of secondary IDH mutations have been observed (Intlekofer et al. 2018; Choe et al. 2020). Relevant to our case, Intlekofer et al. (2018) identified an IDH1 R132-mutated AML patient with appearance of a secondary IDH1 p.S280F variant after ivosidenib therapy. A subsequent study demonstrated the emergence of several secondary IDH1 mutations in ivosidenib-treated IDH1 R132-mutation-bearing patients, including the P.S280F variant, corresponding with elevated serum 2-HG levels and a loss of ivosidenib binding to the p.R132H/p.S280F double mutation carrying IDH1 protein (Choe et al. 2020).

The crystal structure of IDH1 p.R132H was recently solved in association with NADPH and the new pan-IDH inhibitor, AG-881 (Ma and Yun 2018). AG-881 was shown to bind to mutant IDH1 in the same allosteric pocket as ivosidenib. This caused steric hindrance within the substrate binding site as a result of the bent and displaced a10 helixes, which is thought to account for the inhibitory effect of this compound (Ma and Yun 2018). In our own structural model, we first replaced SER280 with phenylalanine and observed its effect on the interaction of mutant IDH1 p.R132 with AG-881. Similar to the finding seen using the model of Choe et al. (2020), the phenylalanine substitution resulted in significant steric clashes, which are predicted to prevent the binding of AG-881 to IDH1 (Fig. 2A). We then replaced AG-881 with ivosidenib and modeled both the IDH1 p.R132H mutant alone and alongside the p.R132H/p.S280F double-mutant variant. The resulting pose was predicted to be even more sterically hindered by the phenylalanine substitution than AG-881, because it also affected the hydrogen bonds present with SER280 (Fig. 2B). Thus, phenylalanine substitution-induced conformational changes affected the allosteric pocket's plasticity and interfered with binding of both inhibitors in different ways. Based on these findings, it is likely that switching to pan-IDH inhibitor therapy (with AG-881) would not be able to revert clinical resistance in IDH1 p.R132H/p.S280F-bearing AML patients, necessitating alternative therapy.

Importantly, this discovery was made possible by switching from an amplicon-based, hotspot-targeting NGS assay to a newly validated, complete-exon hybrid-capture NGS platform. This new assay enabled us to identify the second-site IDH1 mutation located outside of the targeted hotspot IDH1 region. This questions the usefulness of following patients on targeted therapy with hotspot NGS panels. Specifically, this case clearly demonstrates that IDH1 mutant-bearing AML patients receiving ivosidenib therapy would benefit from longitudinal full-exon IDH1 gene sequencing for the detection of emerging resistance variants. Further studies should determine whether early detection of such mutations could be used to modify or alternate therapeutic regimens for more personalized care and improved outcomes. 
COLD SPRING HARBOR Molecular Case Studies
Assessment of acquired IDH1 inhibitor resistance

\section{METHODS}

\section{Clinical Specimens}

The patient was monitored and/or treated at the University of Minnesota Medical Center (UMMC) between May 2016 and May 2019. Bone marrow and/or peripheral blood samples were collected at the time of initial UMMC assessment, longitudinally during therapy, and at relapse after IDH1 inhibitor therapy.

\section{Specimen Processing and Morphologic Assessment}

Bone marrow core biopsy specimens were fixed in acetic zinc formalin (AZF) fixative for at least $1 \mathrm{~h}$ and then placed into decalcification solution (Decal Stat, Decal Chemical Corp.) for $2 \mathrm{~h}$ prior to processing. The specimens were then processed using the automated tissue processors Leica ASP300S and Leica Peloris II (Leica Biosystems Division of Leica Microsystems Inc.). Three micron-section tissue slides were cut from the processed paraffin blocks and stained with hematoxylin and eosin (H\&E) using H\&E automated stainers (Sakura Finetek USA, Inc.; Leica Biosystems). Morphologic assessment of the H\&E-stained bone marrow core biopsy specimens was performed by a board-certified hematopathologist.

\section{Amplicon-Based NGS Testing}

Genomic DNA was extracted from patient bone marrow aspirate samples using the QIAGEN DNeasy blood and tissue kit per manufacturer's instructions (QIAGEN), and sequencing libraries were prepared using an amplicon-based target enrichment method for mutational hotspots on the Fluidigm Biomark Access Array System, as previously described (Henzler et al. 2018). The enriched DNA libraries were sequenced on an Illumina MiSeq instrument (version 3 chemistry, 300-bp paired-end reads; Illumina, Inc.). FASTO files were processed through a custom designed bioinformatics pipeline, termed Scanlndel (Yang et al. 2015). Variant call files (vcfs) were filtered to remove subthreshold calls with $<500 \times$ coverage and/or variant allele frequency (VAF) less than defined, validated thresholds ranging from $1 \%-10 \%$, dependent on the type of mutation, as follows: $5 \%$ for single-nucleotide variants (SNVs) of high clinical utility (list available upon request); $10 \%$ for other $\mathrm{SNV}$; $1 \%$ for insertion-deletion mutations < $3 \mathrm{bp}$; and $5 \%$ for insertion-deletion mutations 3 bp or similar. Clinically relevant mutations from this VAF were annotated by a board-certified molecular genetic pathologist with Genelnsight (Sunquest, Inc.) or GenomOncology software (GenomOncology) and reported. Sequenced regions (i.e., mutational hotspot regions, consisting of indicated exons) of the clinically ordered gene set for this patient were as follows: FLT3 (NM_004119.2): 14-15; IDH1 (NM_005896.2): 4; IDH2 (NM_002168.2): 4; KIT (NM_000222.2): 8-13, 17; KRAS (NM_033360.3): 2-3; NPM1 (NM_002520.4): 11; NRAS (NM_002524.4): 2-3; PDGFRA (NM_006206.4): 12, 14, 18; TP53 (NM_000546.5): 2-11; WT1 (NM_024426.4): 7, 9. The sequencing coverage table for individual genes can be found in the Supplemental Information for each test (Supplemental Table S2).

\section{Hybrid-Capture-Based NGS Testing}

Genomic DNA was extracted from patient bone marrow aspirate samples as stated above and quantified using the Qubit 2.0 fluorometer (ThermoFisher Scientific). Library preparation was carried out by tagmentation following Nextera protocols (Nextera Flex library preparation, Illumina, Inc.). Target enrichment was performed by hybrid capture using vendor-customized baits (Integrated DNA Technologies) and Illumina Rapid capture reagents per manufacturer's protocol. The enriched libraries were sequenced on an Illumina MiSeq instrument (using version 3 chemistry) to a target of 1.5 million reads per sample. FASTQ files were processed, as described above. Variant call files were filtered to remove subthreshold calls 
with less than $125 \times$ coverage and/or variant allele frequency less than the validated threshold of $5 \%$ for both SNVs and indels. Complete exons were sequenced for the following 68 genes in this patient: ABL1 (NM_005157.4), ALK (NM_004304.4), ASXL1 (NM_015338.5), ATM (NM_000051.3), ATRX (NM_000489.3), BCL6 (NM_001706.4), BCOR (NM_001123385.1), BRAF (NM_004333.4), BTK (NM_000061.2), CALR (NM_004343.3), CARD11 (NM_032415.4), CBL (NM_005188.3), CD79A (NM_001783.3), CD79B (NM_000626.2), CDK6 (NM_001259.6), CDKN2A (NM_000077.4), CDKN2B (NM_004936.3), CEBPA (NM_004364.3), CREBBP (NM_004380.2), CSF3R (NM_156039.3), DNMT3A (NM_022552.4), ETV6 (NM_001987.4), EZH2 (NM_004456.4), FGFR1 (NM_023110.2), FLT3 (NM_004119.2), GATA1 (NM_002049.3), GATA2 (NM_032638.4), HRAS (NM_005343.2), IDH1 (NM_005896.2), IDH2 (NM_002168.2), IKZF1 (NM_006060.4), IL7R (NM_002185.3), JAK1 (NM_002227.2), JAK2 (NM_004972.3), JAK3 (NM_000215.3), KIT (NM_000222.2), KRAS (NM_033360.3), MAP2K1 (NM_002755.3), KMT2A (NM_001197104.1), MPL (NM_005373.2), MYC (NM_002467.4), MYD88 (NM_002468.4), NF1 (NM_001042492.2), NOTCH1 (NM_017617.3), NOTCH2 (NM_024408.3), NPM1 (NM_002520.4), NRAS (NM_002524.4), PDGFRA (NM_006206.4), PDGFRB (NM_002609.3), PHF6 (NM_032458.2), PTPN11 (NM_002834.3), RARA (NM_000964.3), RB1 (NM_000321.2), RUNX1 (NM_001754.4), SETBP1 (NM_015559.2), SF1 (NM_001178030.1), SF3A1 (NM_005877.4), SF3B1 (NM_012433.2), SH2B3 (NM_005475.2), SRSF2 (NM_003016.4), STAT3 (NM_139276.2), TET2 (NM_001127208.2), TP53 (NM_000546.5), TYMS (NM_001071.2), U2AF1 (NM_006758.2), U2AF2 (NM_007279.2), WT1 (NM_024426.4), and ZRSR2 (NM_005089.3). The sequencing coverage table for individual genes can be found in the Supplemental Information for each test (Supplemental Table S2). FASTQ files were processed through a customized bioinformatics pipeline at the Minnesota Supercomputing Institute, including CutAdapt (Martin 2011), PandaSeq (Masella et al. 2012), BWA (Li and Durbin 2009), and FreeBayes (Garrison and Marth 2012). VCF files were uploaded to Genelnsight or GenomOncology software for variant filtering, annotation, and reporting by a board-certified molecular genetic pathologist.

\section{Flow Cytometric Immunophenotyping}

Immunophenotyping assays were performed by UMMC, Fairview Clinical Laboratories for standard clinical care, using a BD FACSCanto 10-color flow cytometer (Becton, Dickinson and Company) and Kaluza analysis software (Beckman Coulter Life Sciences). Bone marrow and peripheral blood samples were processed using a stain/lyse protocol combined with a lyse/stain protocol. Cell concentrations were adjusted to ensure optimal antibody staining and the cells were washed before acquisition. Viability was assessed using 7AAD. At follow-up, 10-color analyses were performed for the following antigens: CD3, CD7, CD10, CD11b, CD13, CD14, CD15, CD16, CD19, CD33, CD34, CD38, CD45, CD56, CD64, CD117, and HLA-DR. Cells were gated to isolate populations (forward scatter vs. side scatter and CD45 versus side scatter), to exclude debris (forward scatter vs. side scatter), and to exclude cell doublets (forward scatter height vs. forward scatter width and side scatter height vs. side scatter width). Analyses and reporting were performed by a board-certified hematopathologist.

\section{Structural Modeling}

Ivosidenib was docked to the protein-only structure portion of the cocrystal of IDH1 and AG881 (PDB 6ADG) (Ma and Yun 2018) using smina (Koes et al. 2013) (built February 12, 2019) with the options "-autobox_ligand lig.pdb -seed 0 -flexdist 4 -flexdist_ligand lig.pdb autobox_add 6" where lig.pdb is the extracted AG-881 structure. This treats the side chains of 22 residues with $4 \AA$ of $A G-881$ as flexible, which was necessary in order to successfully 
COLD SPRING HARBOR Molecular Case Studies
Assessment of acquired IDH1 inhibitor resistance
Competing Interest Statement

The authors have declared no competing interest.

Received November 8, 2020; accepted in revised form January 26, 2021. dock ivosidenib into the pocket. The resulting top pose had a predicted affinity of -10.1 $\mathrm{kcal} / \mathrm{mol}$ and is shown in Figure 2.

\section{ADDITIONAL INFORMATION}

\section{Data Deposition and Access}

The consent documentation signed by the patient does not expressly allow submission of full sequencing data (FASTQ, BAM/BAI, VCF) to external data repositories (see below). The variants were deposited in ClinVar (https://www.ncbi.nlm.nih.gov/clinvar/) and can be found under accession numbers VCV000996127 and VCV000375891.

\section{Ethics Statement}

The patient signed the institution-approved, standard consent for clinical diagnostic testing by NGS, including agreement to the opt in/opt out clause for use of genetic information for research purposes. Further verbal consent from the patient was obtained and documented to publish nonidentifying details about the patient's specific diagnosis, clinical test results, and care for advancement of clinical-academic practice. Neither of these consent mechanisms allow for sharing of genetic information beyond that clinically relevant and reported in the manuscript.

\section{Acknowledgments}

We thank William Crowe (University of Rochester) for helping with the preparation of the Supplemental Tables.

\section{Author Contributions}

P.M. conceived and designed the overall project and finalized the manuscript. S.M. performed NGS clinical testing. D.K. modeled protein-inhibitor interactions and performed the docking studies. E.D.W. provided clinical description and context. A.C.N. and S.Y. interpreted the clinical NGS results. Z.N.O. and S.E.H. reviewed and analyzed the data and wrote the manuscript with input from P.M., D.K, A.C.N., and S.Y. All authors read the manuscript and approved its final version.

\section{REFERENCES}

Amatangelo MD, Quek L, Shih A, Stein EM, Roshal M, David MD, Marteyn B, Farnoud NR, de Botton S, Bernard OA, et al. 2017. Enasidenib induces acute myeloid leukemia cell differentiation to promote clinical response. Blood 130: 732-741. doi:10.1182/blood-2017-04-779447

Bullinger L, Döhner K, Döhner H. 2017. Genomics of acute myeloid leukemia diagnosis and pathways. J Clin Oncol 35: 934-946. doi:10.1200/JCO.2016.71.2208

Carbonell D, Suárez-González J, Chicano M, Andrés-Zayas C, Triviño JC, Rodríguez-Macías G, Bastos-Oreiro $M$, Font $P$, Ballesteros $M$, Muñiz $P$, et al. 2019. Next-generation sequencing improves diagnosis, prognosis and clinical management of myeloid neoplasms. Cancers (Basel) 11: 1364. doi:10.3390/cancers11091364

Chaturvedi A, Araujo Cruz MM, Jyotsana N, Sharma A, Yun H, Görlich K, Wichmann M, Schwarzer A, Preller M, Thol F, et al. 2013. Mutant IDH1 promotes leukemogenesis in vivo and can be specifically targeted in human AML. Blood 122: 2877-2887. doi:10.1182/blood-2013-03-491571

Choe S, Wang H, DiNardo CD, Stein EM, de Botton S, Roboz GJ, Altman JK, Mims AS, Watts JM, Pollyea DA, et al. 2020. Molecular mechanisms mediating relapse following ivosidenib monotherapy in IDH1-mutant relapsed or refractory AML. Blood Adv 4: 1894-1905. doi:10.1182/bloodadvances.2020001503

Chotirat S, Thongnoppakhun W, Promsuwicha O, Boonthimat C, Auewarakul CU. 2012. Molecular alterations of isocitrate dehydrogenase 1 and 2 (IDH1 and IDH2) metabolic genes and additional genetic mutations in newly diagnosed acute myeloid leukemia patients. J Hematol Oncol 5: 5. doi:10.1186/1756-8722-5-5 
Dang L, White DW, Gross S, Bennett BD, Bittinger MA, Driggers EM, Fantin VR, Jang HG, Jin S, Keenan MC, et al. 2010. Cancer-associated IDH1 mutations produce 2-hydroxyglutarate. Nature 465: 966. doi:10.1038/ nature09132

Dang L, Yen K, Attar EC. 2016. IDH mutations in cancer and progress toward development of targeted therapeutics. Ann Oncol 27: 599-608. doi:10.1093/annonc/mdw013

DiNardo CD, Wei AH. 2020. How I treat acute myeloid leukemia in the era of new drugs. Blood 135: 85-96. doi:10.1182/blood.2019001239

DiNardo CD, Stein EM, de Botton S, Roboz GJ, Altman JK, Mims AS, Swords R, Collins RH, Mannis GN, Pollyea DA, et al. 2018. Durable remissions with ivosidenib in IDH1-mutated relapsed or refractory AML. N Engl J Med 378: 2386-2398. doi:10.1056/NEJMoa1716984

Forbes SA, Bhamra G, Bamford S, Dawson E, Kok C, Clements J, Menzies A, Teague JW, Futreal PA, Stratton MR. (2008). The Catalogue of Somatic Mutations in Cancer (COSMIC). Curr Protoc Hum Genet Chapter 10: Unit 10.11. doi:10.1002/0471142905.hg1011s57

Garrison E, Marth G. 2012. Haplotype-based variant detection from short-read sequencing. arXiv:1207.3907.

Golub D, lyengar N, Dogra S, Wong T, Bready D, Tang K, Modrek AS, Placantonakis DG. 2019. Mutant isocitrate dehydrogenase inhibitors as targeted cancer therapeutics. Front Oncol 9: 417. doi:10.3389/ fonc.2019.00417

Harding JJ, Lowery MA, Shih AH, Schvartzman JM, Hou S, Famulare C, Patel M, Roshal M, Do RK, Zehir A, et al. 2018. Isoform switching as a mechanism of acquired resistance to mutant isocitrate dehydrogenase inhibition. Cancer Discov 8: 1540-1547. doi:10.1158/2159-8290.CD-18-0877

Henzler C, Schomaker M, Yang R, Lambert AP, LaRue R, Kincaid R, Beckman K, Kemmer T, Wilson J, Yohe S et al. 2018. Optimization of a microfluidics-based next generation sequencing assay for clinical oncology diagnostics. Ann Transl Med 6: 162. doi:10.21037/atm.2018.05.07

Intlekofer AM, Shih AH, Wang B, Nazir A, Rustenburg AS, Albanese SK, Patel M, Famulare C, Correa FM, Takemoto N, et al. 2018. Acquired resistance to IDH inhibition through trans or cis dimer-interface mutations. Nature 559: 125-129. doi:10.1038/s41586-018-0251-7

Jakob CG, Upadhyay AK, Donner PL, Nicholl E, Addo SN, Qiu W, Ling C, Gopalakrishnan SM, Torrent M, Cepa SP, et al. 2018. Novel modes of inhibition of wild-type isocitrate dehydrogenase 1 (IDH1): direct covalent modification of His315. J Med Chem 61: 6647-6657. doi:10.1021/acs.jmedchem.8b00305

Karczewski KJ, Francioli LC, Tiao G, Cummings BB, Alföldi J, Wang Q, Collins RL, Laricchia KM, Ganna A, Birnbaum DP, et al. 2020. The mutational constraint spectrum quantified from variation in 141,456 humans. Nature 581: 434-443. doi:10.1038/s41586-020-2308-7

King RL, Bagg A. 2017. Molecular malfeasance mediating myeloid malignancies: the genetics of acute myeloid leukemia. Methods Mol Biol 1633: 1-17. doi:10.1007/978-1-4939-7142-8_1

Koes DR, Baumgartner MP, Camacho CJ. 2013. Lessons learned in empirical scoring with smina from the CSAR 2011 benchmarking exercise. J Chem Inf Model 53: 1893-1904. doi:10.1021/ci300604z

Landrum MJ, Lee JM, Benson M, Brown G, Chao C, Chitipiralla S, Gu B, Hart J, Hoffman D, Hoover J, et al. 2016. ClinVar: public archive of interpretations of clinically relevant variants. Nucleic Acids Res 44: D862-D868. doi:10.1093/nar/gkv1222

Levell JR, Caferro T, Chenail G, Dix I, Dooley J, Firestone B, Fortin PD, Giraldes J, Gould T, Growney JD, et al. 2017. Optimization of 3-pyrimidin-4-yl-oxazolidin-2-ones as allosteric and mutant specific inhibitors of IDH1. ACS Med Chem Lett 8: 151-156. doi:10.1021/acsmedchemlett.6b00334

$\mathrm{Li} \mathrm{H}$, Durbin R. 2009. Fast and accurate short read alignment with Burrows-Wheeler transform. Bioinformatics 25: 1754-1760. doi:10.1093/bioinformatics/btp324

Li MM, Datto M, Duncavage EJ, Kulkarni S, Lindeman NI, Roy S, Tsimberidou AM, Vnencak-Jones CL, Wolff DJ, Younes A, et al. 2017. Standards and guidelines for the interpretation and reporting of sequence variants in cancer: a joint consensus recommendation of the Association for Molecular Pathology, American Society of Clinical Oncology, and College of American Pathologists. J Mol Diagn 19: 4-23. doi:10 .1016/j.jmoldx.2016.10.002

Losman JA, Kaelin WG Jr. 2013. What a difference a hydroxyl makes: mutant IDH, (R)-2-hydroxyglutarate, and cancer. Genes Dev 27: 836-852. doi:10.1101/gad.217406.113

Losman JA, Looper RE, Koivunen P, Lee S, Schneider RK, McMahon C, Cowley GS, Root DE, Ebert BL, Kaelin WG Jr. 2013. (R)-2-hydroxyglutarate is sufficient to promote leukemogenesis and its effects are reversible. Science 339: 1621-1625. doi:10.1126/science.1231677

Ma R, Yun CH. 2018. Crystal structures of pan-IDH inhibitor AG-881 in complex with mutant human IDH1 and IDH2. Biochem Biophys Res Commun 503: 2912-2917. doi:10.1016/j.bbrc.2018.08 .068

Martignoles JA, Delhommeau F, Hirsch P. 2018. Genetic hierarchy of acute myeloid leukemia: from clonal hematopoiesis to molecular residual disease. Int J Mol Sci 19: 3850. doi:10.3390/ijms19123850

Martin M. 2011. Cutadapt removes adapter sequences from high-throughput sequencing reads. EMBnet Journal 17: 1-12. doi:10.14806/ej.17.1.200 
Masella AP, Bartram AK, Truszkowski JM, Brown DG, Neufeld JD. 2012. PANDAseq: paired-end assembler for illumina sequences. BMC Bioinformatics 13: 31. doi:10.1186/1471-2105-13-31

Medeiros BC, Fathi AT, DiNardo CD, Pollyea DA, Chan SM, Swords R. 2017. Isocitrate dehydrogenase mutations in myeloid malignancies. Leukemia 31: 272-281. doi:10.1038/leu.2016.275

Megías-Vericat JE, Ballesta-López O, Barragán E, Montesinos P. 2019. IDH1-mutated relapsed or refractory AML: current challenges and future prospects. Blood Lymphat Cancer 9: 19-32. doi:10.2147/BLCTT .S177913

Nassereddine S, Lap CJ, Haroun F, Tabbara I. 2017. The role of mutant IDH1 and IDH2 inhibitors in the treatment of acute myeloid leukemia. Ann Hematol 96: 1983-1991. doi:10.1007/s00277-017-3161-0

Ok CY, Loghavi S, Sui D, Wei P, Kanagal-Shamanna R, Yin CC, Zuo Z, Routbort MJ, Tang G, Tang Z, et al. 2019. Persistent IDH1/2 mutations in remission can predict relapse in patients with acute myeloid leukemia. Haematologica 104: 305-311. doi:10.3324/haematol.2018.191148

Quek L, David MD, Kennedy A, Metzner M, Amatangelo M, Shih A, Stoilova B, Quivoron C, Heiblig M, Willekens $C$, et al. 2018. Clonal heterogeneity of acute myeloid leukemia treated with the IDH2 inhibitor enasidenib. Nat Med 24: 1167-1177. doi:10.1038/s41591-018-0115-6

Tommasini-Ghelfi S, Murnan K, Kouri FM, Mahajan AS, May JL, Stegh AH. 2019. Cancer-associated mutation and beyond: the emerging biology of isocitrate dehydrogenases in human disease. Sci Adv 5: eaaw4543. doi:10.1126/sciadv.aaw4543

Upadhyay VA, Brunner AM, Fathi AT. 2017. Isocitrate dehydrogenase (IDH) inhibition as treatment of myeloid malignancies: progress and future directions. Pharmacol Ther 177: 123-128. doi:10.1016/j.pharmthera 2017.03.003

Waitkus MS, Diplas BH, Yan H. 2018. Biological role and therapeutic potential of IDH mutations in cancer. Cancer Cell 34: 186-195. doi:10.1016/j.ccell.2018.04.011

Wang F, Travins J, DeLaBarre B, Penard-Lacronique V, Schalm S, Hansen E, Straley K, Kernytsky A, Liu W, Gliser $\mathrm{C}$, et al. 2013. Targeted inhibition of mutant IDH2 in leukemia cells induces cellular differentiation. Science 340: 622-626. doi:10.1126/science.1234769

Winer ES, Stone RM. 2019. Novel therapy in acute myeloid leukemia (AML): moving toward targeted approaches. Ther Adv Hematol 10: 2040620719860645. doi:10.1177/2040620719860645

Xie X, Baird D, Bowen K, Capka V, Chen J, Chenail G, Cho Y, Dooley J, Farsidjani A, Fortin P, et al. 2017. Allosteric mutant IDH1 inhibitors reveal mechanisms for IDH1 mutant and isoform selectivity. Structure 25: 506-513. doi:10.1016/j.str.2016.12.017

Yang R, Nelson AC, Henzler C, Thyagarajan B, Silverstein KA. 2015. Scanlndel: a hybrid framework for indel detection via gapped alignment, split reads and de novo assembly. Genome Med 7: 127. doi:10.1186/ s13073-015-0251-2

Yen K, Travins J, Wang F, David MD, Artin E, Straley K, Padyana A, Gross S, DeLaBarre B, Tobin E, et al. 2017. AG-221, a first-in-class therapy targeting acute myeloid leukemia harboring oncogenic IDH2 mutations. Cancer Discov 7: 478-493. doi:10.1158/2159-8290.CD-16-1034

Yoshimi A, Lin KT, Wiseman DH, Rahman MA, Pastore A, Wang B, Lee SC, Micol JB, Zhang XJ, de Botton S, et al. 2019. Coordinated alterations in RNA splicing and epigenetic regulation drive leukaemogenesis. Nature 574: 273-277. doi:10.1038/s41586-019-1618-0 


\title{
COLD SPRING HARBOR Molecular Case Studies
}

\section{Assessing acquired resistance to IDH1 inhibitor therapy by full-exon IDH1 sequencing and structural modeling}

\author{
Zoltán N. Oltvai, Susan E. Harley, David Koes, et al.
}

Cold Spring Harb Mol Case Stud 2021, 7: a006007

Access the most recent version at doi: $10.1101 / \mathrm{mcs} . a 006007$
Supplementary http://molecularcasestudies.cshlp.org/content/suppl/2021/04/06/mcs.a006007.D Material C1
References This article cites 43 articles, 9 of which can be accessed free at: http://molecularcasestudies.cshlp.org/content/7/2/a006007.full.html\#ref-list-1
License This article is distributed under the terms of the Creative Commons Attribution-NonCommercial License, which permits reuse and redistribution, except for commercial purposes, provided that the original author and source are credited.
Email Alerting Receive free email alerts when new articles cite this article - sign up in the box at the Service top right corner of the article or click here.

